\title{
DEFICIÊNCIAS NUTRICIONAIS DE MACRONUTRIENTES E SÓDIO EM MUDAS DE PUPUNHEIRA ${ }^{1}$
}

\author{
ANTONIO RODRIGUES FERNANDES², GILSON SERGIO BASTOS DE MATOS ${ }^{3}$, \\ JANICE GUEDES DE CARVALHO ${ }^{+}$
}

RESUMO - O cultivo da pupunheira (Bactris gasipaes Kunth) tem aumentado em função da crescente demanda por seus produtos, entretanto são reduzidos os estudos, bem como o conhecimento sobre as exigências de nutrientes da espécie. O objetivo foi avaliar os sintomas de deficiência nutricional, a produção de matéria seca, o teor e o acúmulo de nutrientes em mudas de pupunheira, em razão da omissão de macronutrientes e $\mathrm{Na}$. O experimento foi realizado em casa de vegetação utilizando mudas em vasos distribuídos em delineamento de blocos casualizados. Os tratamentos foram: solução nutritiva completa; omissão de N, P, K, Ca, Mg, S e Na. As omissões promoveram alterações morfológicas na parte aérea e nas raízes caracterizadas por sintomas visuais facilmente interpretáveis. Os elementos N, P e K foram os mais limitantes, já $\mathrm{S}$ e $\mathrm{Na}$ os menos limitantes à produção de matéria seca das folhas, caule, raízes, parte aérea $\mathrm{e}$ total da planta. A relação da matéria seca da parte aérea/raiz foi $\mathrm{K}>\mathrm{Mg}>\mathrm{Ca}=\mathrm{P}$ e $\mathrm{P}=$ completo $=\mathrm{S}=\mathrm{Na}>\mathrm{N}$, e o crescimento relativo foi decrescente no sentido Completo $>\mathrm{Na}>\mathrm{S}>\mathrm{Ca}>\mathrm{Mg}>\mathrm{K}>\mathrm{P}>\mathrm{N}$. Os teores e acúmulos de nutrientes seguiram a ordem predominante $\mathrm{N}>\mathrm{K}>\mathrm{Ca}>\mathrm{P}>\mathrm{Mg}>\mathrm{S}>\mathrm{Na}$. As omissões causaram sintomas típicos aos observados em outras espécies. A matéria seca e o crescimento relativo foram mais limitados pelo $\mathrm{N}, \mathrm{P}$ e $\mathrm{K}$, sendo que o $\mathrm{K}, \mathrm{Mg}$, Ca e $\mathrm{P}$ restringiram mais as raízes, enquanto os outros nutrientes limitaram mais a parte aérea. A ausência de Na reduziu o crescimento relativo em 10,6\%, o que demonstra a importância do elemento para a pupunheira. Os nutrientes em maiores quantidades na espécie foram o $\mathrm{N}, \mathrm{K}$ e Ca.

Palavras-chave: Bactris gasipaes, matéria seca, teor e acúmulo de nutrientes, omissão de nutrientes.

\section{NUTRITIONAL DEFICIENCIES OF MACRONUTRIENTS AND SODIUM IN PEACH PALM SEEDLINGS}

\begin{abstract}
The peach palm (Bactrisgasipaes Kunth) cultivation has increased according to increasing demand of its products; however there are few studies, as well as knowledge about nutritional requirement of this specie. The objective was to evaluate the nutritional symptoms deficiencies, dry matter production, nutrient content and accumulation in peach palm seedlings, according to macronutrients and $\mathrm{Na}$ omission. The experiment was realized in greenhouse in randomized blocks design. The treatments were: complete nutrient solution; omission of $\mathrm{N}, \mathrm{P}, \mathrm{K}, \mathrm{Ca}, \mathrm{Mg}, \mathrm{S}$ and $\mathrm{Na}$. The omissions promoted morphological alterations in shoots and roots characterized by visual symptoms easily interpretable. The elements $\mathrm{N}, \mathrm{P}$ and $\mathrm{K}$ were the most limiting and the $\mathrm{S}$ and $\mathrm{Na}$ were the least limiting to leaves, stem, roots, shoots and total dry matter. The shoot/root relationship was $\mathrm{K}>\mathrm{Mg}>\mathrm{Ca}=\mathrm{P}$ and $\mathrm{P}=$ complete $=\mathrm{S}=\mathrm{Na}>\mathrm{N}$ and the relative growth was complete $>\mathrm{Na}>\mathrm{S}>\mathrm{Ca}>\mathrm{Mg}>\mathrm{K}>\mathrm{P}>\mathrm{N}$. The nutrient contents and accumulations follow mainly the sequence $\mathrm{N}>\mathrm{K}>\mathrm{Ca}>\mathrm{P}>\mathrm{Mg}>\mathrm{S}>\mathrm{Na}$. The omissions caused symptoms typical to those observed in other species. The dry matter and relative growth were most limiting by $\mathrm{N}, \mathrm{P}$ and $\mathrm{K}$. The $\mathrm{K}, \mathrm{Mg}, \mathrm{Ca}$ and $\mathrm{P}$ were most limiting to roots and others nutrients were most limiting to shoots. The $\mathrm{Na}$ was important to peach palm, in the absence of $\mathrm{Na}$ of growth on reduced $10.6 \%$. The nutrients in higher amounts in the specie are $\mathrm{N}, \mathrm{K}$ and $\mathrm{Ca}$. Index terms: Bactrisgasipaes Kunth, dry matter, nutrients content and accumulation, the nutrient omission.
\end{abstract}

\footnotetext{
1(Trabalho 045-13). Recebido em: 04-01-2013. Aceito para publicação em: 27-09-2013.

${ }^{2}$ Eng. ${ }^{\circ}$. Agr. ${ }^{\circ}$ Prof. Dr. do Instituto de Ciências Agrárias da Universidade Federal Rural da Amazônia, Av. Presidente Tancredo Neves, 2501,CEP 66077-530, Belém. E-mail: antonio.fernandes@ufra.edu.br

${ }^{3}$ Eng. ${ }^{\circ}$ Agr. ${ }^{\circ}$ Doutorando do Programa de Pós-Graduação em Agronomia da Universidade Federal Rural da Amazônia, Av. Presidente Tancredo Neves, 2501, CEP 66077-530, Belém, PA, Brasil. E-mail: gilsonsbm@yahoo.com.br

TIn memorian
} 


\section{INTRODUÇAO}

A pupunheira é uma palmeira nativa da região tropical das Américas, sendo proveniente da bacia amazônica, ocorrendo em todos os estados que a compõem (DEENIK et al., 2000). Seus atrativos econômicos estão relacionados ao fruto, rico em vitamina $\mathrm{A}$ e energia, destinado à alimentação humana e animal, assim como à extração de óleo. $\mathrm{O}$ uso mais recente e de maior importância econômica da espécie é para extração de palmito, que tem difundido a cultura para as regiões Nordeste, Sudeste e Sul do Brasil (YUYAMA et al., 2005).

A espécie é precoce e possui elevada capacidade de perfilhamento, possibilitando até dois cortes de palmito por touceira no ano, o que confere superioridade de produção em relação ao palmito do açaí (Euterpe oleracea) e da juçara (Euterpe edulis). Tais espécies são responsáveis por $99 \%$ do palmito do mercado brasileiro, cuja exploração é predominantemente extrativista.

Apesar da expansão dos plantios de pupunheira, poucos são os trabalhos a respeito da fertilidade do solo e da nutrição mineral das plantas (DEENIK et al., 2000; FERNANDES et al., 2002; YUYUAMA et al., 2005). Em função disso, os dados existentes sobre a nutrição da pupunheira são insuficientes para definir o manejo e as exigências nutricionais da cultura.

A técnica do elemento faltante em soluções nutritivas avalia a exigência nutricional das culturas e constitui uma ferramenta eficiente para fornecer informações qualitativas sobre os nutrientes que podem limitar o desenvolvimento das plantas (LAVIOLA; DIAS, 2008; MIRANDA et al., 2010).

Em estudo realizado no Amazonas, utilizando a técnica do elemento faltante com mudas de pupunheira, o $\mathrm{N}$ foi o nutriente mais limitante ao desenvolvimento, seguido por K e Ca (SILVA; FALCÃO, 2002). Entretanto, são inexistentes trabalhos relacionando as limitações aos acúmulos de nutrientes, bem como ao crescimento, utilizando a solução indicada para espécies da família Arecaceae, desenvolvida por Dufour et al. (1978). Além disso, pesquisas têm constatado a importância do elemento Na para o desenvolvimento de mudas de pupunheiras (FERNANDES; CARVALHO, 2001; FERNANDES et al., 2002) sem indicar os sintomas foliares de deficiências, tampouco, informar teores adequados e quantidades acumuladas desse elemento nas partes das plantas.

$\mathrm{O} \mathrm{Na}$ é um elemento considerado benéfico, principalmente para espécies natrofílicas e halófitas, podendo substituir o $\mathrm{K}$ no vacúolo, na ativação de diversas enzimas, influenciando a osmose da membrana e regulando a mobilidade estomática (PILON-SMITS et al., 2009). Esse nutriente também estimula a expansão celular e, em algumas plantas C4 e CAM, tem sido considerado um elemento essencial, e em condição de limitação do suprimento de $\mathrm{Na}$, as plantas podem responder com decréscimo ao desenvolvimento, clorose e necrose dos ápices foliares e das margens (SUBBARAO et al., 2003).

Para algumas espécies de Arecaceae, foi constatada resistência à salinidade ocasionada por $\mathrm{Na}$, bem como esse elemento foi relacionado ao aumento do crescimento e da produção (FERNANDES; CARVALHO, 2001; FERNANDES et al., 2002; SOUSA et al., 2004). Trabalhos que relacionem o desenvolvimento da planta com a presença ou a ausência de $\mathrm{Na}$ em palmeiras podem contribuir para a definição de seu papel sobre a nutrição mineral dessas culturas.

O objetivo foi avaliar o efeito da omissão de macronutrientes e de $\mathrm{Na}$ a partir dos sintomas de deficiências nutricionais, produção de matéria seca, teor e acúmulo de nutrientes em pupunheira.

\section{MATERIAL E MÉTODOS}

O experimento foi conduzido em casa de vegetação do Departamento de Ciência do Solo, da Universidade Federal de Lavras, utilizando-se de mudas de pupunheira (Bactris gasipae Kunth) cultivadas em solução nutritiva.

As sementes foram oriundas do Peru, de plantas sem espinhos. Foram germinadas em bandejas plásticas contendo vermiculita. Após 30 dias da germinação, foram colocadas em bandejas coletivas com capacidade para 36 litros de solução de Dufour et al. (1978), a 1/4 da força iônica, durante 30 dias, e $1 / 2$ força iônica, durante mais 15 dias. Nesse momento, com cerca de três folhas e $20 \mathrm{~cm}$, as mudas foram transferidas para vasos com capacidade de $9 \mathrm{~L}$, de acordo com os tratamentos, permanecendo por cinco meses. As soluções foram renovadas a cada 20 dias, nos primeiros dois meses, e, a partir daí, a cada 15 dias. As soluções foram mantidas sob aeração constante durante o período experimental (5 meses), e o volume, mantido pela reposição diária de água deionizada. A medição do $\mathrm{pH}$ era realizada diariamente e, quando abaixava de 5,5, a solução era trocada.

O delineamento experimental foi em blocos casualizados, com oito tratamentos e três repetições, adotando-se a técnica do elemento faltante. Os tratamentos foram: completo (macro e micronutrientes); omissão de N; P; K; Ca, Mg, S e 
Na. As unidades experimentais foram constituídas por vasos contendo uma planta. A solução nutritiva básica (DUFOUR et al., 1978) para macronutrientes foi composta de: $\mathrm{NO}_{3}=8 ; \mathrm{NH}_{4}=2 ; \mathrm{P}=1 ; \mathrm{K}=2$; $\mathrm{C}$. $=2 ; \mathrm{Mg}=1,5 ; \mathrm{S}=1 ; \mathrm{Na}=1,0$ e $\mathrm{Cl}=0,5 \mathrm{mmol}$ $\mathrm{L}$, que correspondeu ao tratamento-controle. As concentrações dos macronutrientes foram as mesmas para os demais tratamentos, com a omissão individual de nutrientes, tendo-se o cuidado para manter o balanço iônico. Os sais que forneceram os nutrientes foram: $\mathrm{Ca}\left(\mathrm{NO}_{3}\right)_{2} ; \mathrm{KNO}_{3} ; \mathrm{NH}_{4} \mathrm{NO}_{3}$; $\mathrm{NaNO}_{3} ; \mathrm{Mg}\left(\mathrm{NO}_{3}\right)_{2} ;\left(\mathrm{NH}_{4}\right)_{2} \mathrm{SO}_{4} ; \mathrm{NaH}_{2} \mathrm{PO}_{4} ; \mathrm{KH}_{2} \mathrm{PO}_{4}$; $\mathrm{KCl} ; \mathrm{MgSO}_{4} ; \mathrm{MgCl}_{2}$ e $\mathrm{Ca}\left(\mathrm{S}_{-} \mathrm{P}_{4}\right)$. As concentrações dos micronutrientes em $\mathrm{mg} \mathrm{L}$ e seus respectivos sais foram: $\mathrm{B}=0,20\left(\mathrm{H}_{3} \mathrm{BO}_{4}\right) ; \mathrm{Cu}=0,05\left(\mathrm{CuSO}_{4} \cdot 5 \mathrm{H}_{2} \mathrm{O}\right)$; $\mathrm{Fe}=3,00($ FeEDTA $) ; \mathrm{Mn}=0,35\left(\mathrm{MnSO}_{4} \cdot \mathrm{H}_{2} \mathrm{O}\right)$; $\mathrm{Mo}=0,02\left(\left(\mathrm{NH}_{4}\right) 6 \mathrm{Mo}_{7} \mathrm{O} \cdot 4 \mathrm{H}_{2} \mathrm{O}\right)$ e $\mathrm{Zn}=0,05$ $\left(\mathrm{ZnSO}_{4} .7 \mathrm{H}_{2} \mathrm{O}\right)$.

Durante o período experimental, houve monitoramento diário dos sintomas visuais de deficiência. Tais sintomas foram fotografados por ocasião da colheita das plantas, ocasião em que todas as plantas apresentavam algum tipo de sintoma, e mesmo aquelas que apresentaram os primeiros sintomas, permaneceram vivas.

Após a colheita, o material vegetal foi separado em folha, caule e raiz, sendo lavadas com água destilada, em seguida, secas em estufa de circulação forçada de ar, à temperatura de 65$70{ }^{\circ} \mathrm{C}$, até que apresentassem peso constante. $\mathrm{O}$ material vegetal foi pesado em balança de precisão para a obtenção da massa de matéria seca de cada componente, para posterior determinação da relação parte aérea/raiz (PA/R), e crescimento relativo (CR). Calculou-se a relação $\mathrm{PA} / \mathrm{R}$, dividindo-se a massa da matéria seca da parte aérea (folhas + caule) pela massa da matéria seca da raiz. O CR foi determinado utilizando a seguinte formula: $\mathrm{R}=$ (matéria seca dos tratamentos com omissão individual do nutriente/ matéria seca do controle) x 100.

O material seco foi moído em moinho tipo Willye e submetido à digestão nitroperclórica para a determinação dos teores de $\mathrm{N}, \mathrm{P}, \mathrm{K}, \mathrm{Ca}, \mathrm{Mg}, \mathrm{S}$ e $\mathrm{Na}$ nos tecidos (MALAVOLTA et al., 2006). Para a determinação dos acúmulos dos nutrientes, multiplicou-se o teor em $\mathrm{g} \mathrm{kg}^{-1}$, de cada nutriente, pelos valores da matéria seca em cada parte da planta.

Os resultados referentes às variáveis avaliadas foram submetidos à análise de variância (ANOVA), teste $\mathrm{F}$, e, quando observados efeitos significativos para os tratamentos, realizou-se o teste de Tukey ( $p$ $\leq 0,05)$ para a comparação das médias.

\section{RESULTADOS E DISCUSSÃO}

Os sintomas de deficiência apareceram na seguinte ordem: $\mathrm{N}, \mathrm{K}, \mathrm{Mg}, \mathrm{P}, \mathrm{S}, \mathrm{Ca}$ e Na. O sintoma de deficiência de $\mathrm{N}$ apareceu aos 35 dias após a aplicação dos tratamentos. A deficiência reduziu o crescimento das mudas de pupunheira quando comparadas ao tratamento completo (Figura 1A). No início, ocorreu clorose nas folhas mais velhas que, com o tempo, tornou-se generalizada, e todas as folhas apresentaram-se cloróticas ao final do período de cultivo. Esses resultados também concordam com os reportados por Deenik et al. (2000) para a pupunheira, sendo que esses autores citam a clorose ocorrendo especialmente nas extremidades das folhas mais maduras. Em mudas de pupunheira com omissão de N, também foi observada clorose, inicialmente nas folhas mais velhas, aos 60 dias de cultivo, seguida de necrose que se generalizou para toda a planta (SILVA; FALCÃO, 2002). A manifestação dos sintomas em tempos diferentes pode estar relacionada ao tipo de solução e ao período de aclimatação das mudas; além disso, ao tamanho das mudas, cujas reservas nutricionais variam com a idade.

$\mathrm{O}$ amarelecimento inicialmente das folhas mais velhas das plantas sob deficiência de $\mathrm{N}$ é devido à redistribuição dos aminoácidos e à proteólise das proteínas, resultando no colapso dos cloroplastos e no decréscimo do teor de clorofila (HORTENSTEINER; FELLER, 2002). Além disso, causa alterações na atividade das enzimas da fase bioquímica da fotossíntese, do fotossistema II, bem como das enzimas do metabolismo dos carboidratos e do nitrogênio (PAUL; DRISCOLL, 1997), como a ribulose bifosfato carboxilase (RUBISCO), o que leva à menor taxa fotossintética e consequente redução do crescimento.

A omissão de $\mathrm{P}$ causou sintomas visíveis aos 55 dias após o transplantio, caracterizando redução do crescimento das plantas e aparecimento de coloração parda à púrpura das folhas, que evoluíram das bordas para o ráquis e das folhas mais velhas para as mais novas. Em estágio avançado, as folhas mais velhas apresentaram clorose por todo o limbo foliar e necrose, iniciada nos bordos das folhas em direção à nervura central, levando à senescência e ao aparecimento de manchas amarronzadas no limbo (Figura 1B). O P atua na síntese de RNA e DNA, é elemento estrutural de nucleotídeos e fosfolipídios, e atua na transferência de energia por meio de ATP, PPi e NADPH (CUNHA et al., 2009), e com a deficiência há uma redução na produção e no transporte de energia, levando a um menor crescimento.

O suprimento limitado promove a mobilização 
de $\mathrm{P}$ das folhas mais velhas paras as folhas jovens e as raízes (SCHACHTMAN et al., 1998), acelerando a degradação das macromoléculas, culminando na necrose das folhas. Já a coloração que varia de marrom a roxa é causada pelo acúmulo de açúcares nos tecidos, que por sua vez sintetizam pigmentos escuros, às antocianinas (KARTHIKEYAN et al., 2007). Em plantas de açaizeiro, espécie da mesma família da pupunheira, cultivadas em solo com limitação de $\mathrm{P}$, foi observada $50 \%$ de redução do tamanho das plantas em relação ao tratamento completo (VIÉGAS et al., 2009).

Plantas de pupunheira cultivadas com baixos níveis de $\mathrm{P}$, em condições de campo, restringiram a produção de palmito e estirpe tenra (YUYAMA et al., 2005).

O sistema radicular das plantas deficientes em $\mathrm{P}$ apresentou escurecimento, maior comprimento das raízes primárias e menor quantidade de radicelas, o que levou a um menor volume total das raízes (Figura 1C). A limitação do suprimento de $\mathrm{P}$ altera a estabilidade das membranas e o metabolismo vegetal (CUNHA et al., 2009), o que levou à redução do volume radicular. Tem sido reportado na literatura que a deficiência de P modifica a arquitetura do sistema radicular a partir da redução do crescimento de raízes primárias e o estímulo no aumento das raízes secundárias, que tem maior potencial de absorção (LÓPEZ-BUCIO et al., 2003). Entretanto, a resposta das raízes à deficiência de $\mathrm{P}$ depende de cada cultura ou genótipo, sendo que algumas espécies de plantas respondem à deficiência de $\mathrm{P}$ com redução da densidade de raízes laterais ou secundárias (FITA et al., 2011).

Verificou-se redução do tamanho das mudas com omissão de $\mathrm{K}$ em relação ao tratamento completo (Figura 1D). No início da manifestação dos sintomas (38 dias), ocorreu clorose nas folhas mais velhas, e posteriormente necrose, que evoluiu das margens para o centro do limbo foliar, bem como das folhas mais velhas para as mais novas. Esses sintomas assemelham-se aos descritos por Deenik et al. (2000) e Silva e Falcão (2002) para a pupunheira. A rápida redistribuição de $\mathrm{K}$ na planta, das folhas mais maduras para as mais jovens, acelera o amarelecimento das folhas mais velhas (MARQUES et al., 2004) e necroses nas bordas do limbo, as quais estão associadas ao acúmulo da enzima putrescina, produzida na ausência de potássio. O K atua na manutenção do turgor celular e na regulação osmótica estomática, garantindo um regime hídrico favorável (CUNHA et al., 2009), influencia também no transporte e no armazenamento de carboidratos, síntese de proteínas, atividade de cerca de 50 tipos de enzimas e síntese de amido foliar (ARMENGUARD et al., 2009). A deficiência de K, provavelmente, ocasionou redução do transporte de água via transpiração e pressão radicular, levando ao murchamento dos tecidos vegetais com maior taxa de transpiração, resultando em necrose (KANAI et al., 2007).

Nos sintomas de deficiência de cálcio, constatou-se amarelecimento das pontas e margens das folhas mais novas com perceptível curvamento do ápice, semelhante a ganchos, e notórias ondulações no limbo, ficando visíveis próximos aos 120 dias (Figura 1E). As raízes apresentaram menor tamanho que no tratamento completo, poucas ramificações e coloração amarelo-escura (Figura 1F). Sintomas semelhantes foram observados em plantas de açaí (VIÉGAS et al., 2009).

O Ca é considerado um elemento pouco móvel de seu local de residência no vegetal, assim seus sintomas de deficiência manifestam-se nos tecidos em formação (WHITE; BROALEY, 2003). A imobilidade desse nutriente está relacionada ao não carregamento do mesmo nos elementos de tubo crivado e, por ser mensageiro secundário nas respostas do vegetal a sinais ambientais e hormonais por meio do complexo Ca-proteína calmodulina, sua concentração é mantida bastante estável no interior das células (HETHERINGTON; BROWNLEE, 2004). Além disso, dado seu requerimento à formação das estruturas pécticas da parede celular que surge entre as células recém-formadas, com a falta de $\mathrm{Ca}$, os tecidos meristemáticos jovens, apicais e laterais, ficam cloróticos, necróticos e deformados (WHITE; BROALEY, 2003).

Nas raízes, a falta de $\mathrm{Ca}$ interrompe seu alongamento e a formação das paredes celulares, que desfavorece a estruturação dos primórdios radiculares (BELLAMINE et al., 1998). Sua carência influencia no aparecimento de núcleos poliploides e/ou constritos, células binucleadas e divisões amitóticas, fatores que resultam no escurecimento, seguido por morte do sistema radicular (CUNHA et al., 2009). A rigidez da parede celular é influenciada pelo Ca ligado a substâncias pécticas; por outro lado, para que ocorra o alongamento da célula, é necessário afrouxamento das paredes celulares, que é induzido por auxina, hormônio regulado pelo cálcio, e realocação do Ca nas cadeias pécticas (CUNHA et al., 2009).

A deficiência de $\mathrm{Mg}$ foi caracterizada pela redução visual do crescimento da planta em comparação ao tratamento completo. Nas folhas mais velhas, ocorreram manchas cloróticas entre as nervuras (Figuras $1 \mathrm{G} \mathrm{e} \mathrm{1H).} \mathrm{Os} \mathrm{sintomas} \mathrm{ficaram} \mathrm{visíveis} \mathrm{aos}$ 
42 dias depois da omissão do elemento na solução. A clorose internerval é um sintoma característico da deficiência desse nutriente e foi observada de forma semelhante na cultura do açaizeiro (VIÉGAS et al., 2004), e para o maracujazeiro-doce (Passiflora alata) (FREITAS et al., 2011), estando relacionada à fácil redistribuição do $\mathrm{Mg}$ na planta. Diferentemente, plantas de pupunheira no campo e em fase de produção, quando deficientes de $\mathrm{Mg}$, apresentaram amarelecimento das margens dos ápices foliares (ARES et al., 2003). O Mg é constituinte estrutural da clorofila, está relacionado à conformação das proteínas, ativação de enzimas, como a RUBISCO, e fosfoenolpiruvato carboxilase e na transferência de energia através dos substratos ATP-Mg e PPi-Mg (SHAUL, 2002). A clorose internerval, resultante da deficiência de $\mathrm{Mg}$, ocorre devido à clorofila nos feixes vasculares permanecer inalterada durante mais tempo que a clorofila das células entre os feixes (SHAUL, 2002).

As plantas com deficiência de S apresentaram clorose primeiramente nas folhas mais novas; posteriormente, a coloração dessas folhas apresentou tom de vermelho a roxo e os ápices apresentaram sensível retorcimento (Figura 1I e 1J), observado a partir dos 90 dias de omissão do nutriente. Esses sintomas são semelhantes aos encontrados em plantas de açaizeiro (VIÉGAS et al., 2009) e acácia (Acacia holosericea A. Cunn. ex G. Don.) (SARCINELLI et al., 2004). O enxofre é mobilizado em sua maior parte na direção acrópeta pelo xilema, em detrimento da direção basípeta pelo floema; portanto, com a deficiência, os sintomas ocorrem nos tecidos mais novos como os das folhas jovens (HAWKESFORD, 2000). O S constitui diversas enzimas, como a cisteína e a metionina, agentes redutores, aminoácidos, polissacarídeos e sulfatos (ZHAO et al., 1999). A coloração arroxeada observada nas plantas é decorrente do acúmulo de antocianinas causado pela deficiência de S (HAWKESFORD, 2000).

Quando se omitiu o Na, ocorreu redução das mudas em relação ao tratamento completo (Figura $1 \mathrm{~K})$. As folhas apresentaram redução do tamanho, clorose e necrose de suas ponteiras (Figura 1L), quando as mudas atingiram 130 dias de cultivo na solução sem o Na. Sintomas de deficiência de $\mathrm{Na}$ em pupunheira ainda não tinham sido reportados na literatura. Esses resultados são importantes, uma vez que sintomas de deficiência de Na são muito difíceis de serem visualizados, principalmente em solução nutritiva, em função da presença desse elemento na maioria dos reagentes. Entretanto, na planta Atriplex versicaria, na qual o $\mathrm{Na}$ é um elemento essencial, a deficiência leva à clorose e necrose das folhas e à redução do crescimento (SUBBARAO et al.,
2003), tal como verificado no presente trabalho. O $\mathrm{Na}$ tem sido considerado por muitos autores como útil a espécies pertencentes à família Arecaceae devido a favorecer o crescimento e o incremento de produção (FERNANDES; CARVALHO, 2001; FERNANDES et al., 2002). Esses resultados divergem dos encontrados para a cultura do açaizeiro, cujo crescimento da planta não foi afetado pela ausência de $\mathrm{Na}$ (SOUSA et al., 2004). Para algumas plantas C4 e CAM, o Na tem sido considerado um elemento essencial, pois é necessário no transporte de $\mathrm{CO}_{2}$ até às células (SUBBARÃO, 2003).

A matéria seca das folhas, caule, raízes, parte aérea e total foi limitada pela omissão da maioria dos nutrientes, quando comparada ao tratamento completo (Tabela 1). Apesar de provocarem sintomas de deficiências, a ausência de $\mathrm{S}$ e Na não reduziu o crescimento das plantas ( $\mathrm{p}>$ $0,05)$. A matéria seca de todos os compartimentos e a total foram menores quando se omitiu N, P e $\mathrm{K}$ em relação ao tratamento completo. A maior limitação no crescimento das plantas, provocada pela omissão de $\mathrm{N}$, pode ser devida ao maior requerimento do nutriente para a realização dos processos metabólicos, quando comparado a outros nutrientes. Com a deficiência de $\mathrm{N}$, o processo de divisão celular nos pontos de crescimento é retardado (ANANDACOOMARASWAMY et al., 2002), resultando na redução da área foliar e do crescimento da planta. Na cultura do açaizeiro, os nutrientes que mais limitaram a matéria seca das mudas foram o $\mathrm{P}$ seguido por $\mathrm{N}$ e K, quando cultivadas em Latossolo Amarelo textura média (VIEGAS et al., 2004). Isto pode ser justificado devido ao $\mathrm{P}$ naquele solo ser o nutriente mais limitante.

A ordem da relação $\mathrm{PA} / \mathrm{R}$ foi $\mathrm{K}>\mathrm{Mg}>\mathrm{Ca}=\mathrm{Pe}$ $\mathrm{P}=$ Completo $=\mathrm{S}=\mathrm{Na}>\mathrm{N}$ (Tabela 2 ). Ressalta-se que a relação $\mathrm{PA} / \mathrm{R}$ para o $\mathrm{Ca}$ foi maior que no completo. Esse resultado indica que a omissão $\mathrm{K}, \mathrm{Mg}$ e $\mathrm{Ca}$ compromete mais o crescimento do sistema radicular do que a parte aérea em mudas de pupunheira. A deficiência de $\mathrm{K}$ afeta negativamente a formação de novas raízes em função do importante papel no transporte e na acumulação de carboidratos (CUNHA et al., 2009). O Mg atua na ativação de enzimas que agem na respiração, fotossíntese e síntese de ácidos nucleicos (SHAUL, 2002); assim sendo, a omissão compromete a formação de primórdios radiculares (CUNHA et al., 2009), reduzindo o crescimento. A integridade e a estruturação das paredes celulares conferidas pelo Ca são negativamente afetadas pela carência desse nutriente, acarretando na diminuição do alongamento radicular (WHITE; BROALEY, 2003). Para a omissão de $P$, a menor produção de 
matéria seca das raízes pode estar relacionada a sua atuação na síntese de ácidos nucleicos e na estabilidade das membranas (CUNHA et al., 2009).

De forma contrária, a omissão de $\mathrm{N}$ reduziu mais expressivamente a parte aérea, causando a menor relação PA/R (Tabela 1). A limitação do crescimento vegetal pelo $\mathrm{N}$ pode ter transformado as raízes em um forte dreno, e maior quantidade de fotoassimilados foram translocados para as raízes, causando maior limitação ao crescimento da parte aérea (RICHARD-MOLARD et al., 2008). O N foi o nutriente que proporcionou a menor relação $\mathrm{PA} / \mathrm{R}$ na cultura do açaizeiro (VIÉGAS et al., 2009).

A omissão dos macronutrientes e do $\mathrm{Na}$ de forma individual provocou redução do crescimento relativo (CR), cuja ordem decrescente foi: completo $>\mathrm{Na}>\mathrm{S}>\mathrm{Ca}>\mathrm{Mg}>\mathrm{K}>\mathrm{P}>\mathrm{N}$. A menor redução do CR ocorreu com a omissão do Na. Esta redução, embora pequena $(10,6 \%)$, evidencia efeito depressivo no crescimento das mudas de pupunheira pela omissão do Na. Este resultado concorda com os relatos de pesquisas anteriores, que verificaram importância desse elemento na composição nutricional da pupunheira (FERNANDES et al., 2002). O elemento $\mathrm{Na}$ estimula o crescimento por meio do alongamento celular e pode substituir o $\mathrm{K}$ como um soluto osmoticamente ativo (PILONSMITS et al., 2009). A pupunheira desenvolve-se bem na presença de $\mathrm{Na}$, entretanto a quantidade desse elemento não pode exceder $50 \%$ da concentração de $\mathrm{K}$ na solução, havendo nesse caso redução do crescimento da planta (FERNANDES; CARVALHO, 2001). Para mudas de açaizeiro, a adição de $1 \mathrm{mmol}$ de Na no tratamento completo resultou em plantas com maior produção de matéria seca e acúmulo de nutrientes (SOUSA et al., 2004), o que corresponde ao tratamento completo deste estudo. Desta forma, o crescimento relativo das mudas foi reduzido pela omissão de todos os elementos avaliados, sendo menos afetado pela omissão de $\mathrm{Na}$, seguido pelo $\mathrm{S}$ (16\%). Por outro lado, o $\mathrm{N}$ foi o que mais afetou a produção de matéria seca (87\%).

Em solução completa, a sequência decrescente de macronutrientes quanto aos teores nas folhas $\mathrm{e}$ caule seguiu a ordem $\mathrm{N}>\mathrm{K}>\mathrm{Ca}>\mathrm{P}>\mathrm{Mg}>\mathrm{Na}>\mathrm{S}$, e para a raiz a ordem foi $\mathrm{N}>\mathrm{K}>\mathrm{Ca}>\mathrm{P}>\mathrm{Mg}>\mathrm{S}>\mathrm{Na}$ (Tabela 2). Assim, a parte aérea e as raízes apresentaram padrões diferentes quanto aos teores dos nutrientes $\mathrm{S}$ e $\mathrm{Na}$. Esse resultado diferiu do encontrado em trabalho com mudas de pupunheira no Estado do Amazonas, onde foram observados para os macronutrientes a ordem decrescente N, K, Ca, Mg e depois o P (SILVA; FALCÃO, 2002).

Os teores de $\mathrm{Na}$ em plantas crescidas na solução completa foram maiores nos caules, seguidos pelas folhas e raízes. Essa distribuição contraria a relatada por Marques et al. (2004) para um grande número de espécies, ou seja, teores nas folhas $>$ caules $>$ raízes.

A omissão dos nutrientes, quando comparada ao tratamento completo, causou redução no teor daquele que foi omitido, nas folhas, caules e raízes das plantas (Tabela 2). A omissão de $\mathrm{N}$ causou aumento no teor de $\mathrm{P}$ nas folhas e redução nos caules, sendo que nas raízes reduziu os teores de $\mathrm{Ca}$ e $\mathrm{Mg}$. Os aumentos ou a diminuição nos teores de $\mathrm{P}$ e de $\mathrm{Ca}$ e $\mathrm{Mg}$, respectivamente, estão relacionados ao desequilíbrio nutricional na planta. Nas folhas, o teor de $\mathrm{N}$ foi reduzido com a omissão de $\mathrm{S}$. A redução dos teores foliares de N, quando se omitiu o S, está relacionada ao acúmulo de carboidratos, diminuição do teor de açúcares redutores e da síntese de proteínas (HAWKESFORD, 2000), em função da menor conversão de $\mathrm{N}$ para a forma orgânica (PRADO et al., 2007).

Nas folhas das plantas em que houve omissão de $\mathrm{P}$, o teor de $\mathrm{K}$ aumentou, enquanto com a omissão de $\mathrm{K}$ houve aumento nos teores de $\mathrm{N}$ e P (Tabela 2). A omissão de K também aumentou os teores de $\mathrm{Ne} \mathrm{S}$ nas raízes e diminuiu os teores de $\mathrm{Ca}$ e $\mathrm{Mg}$, enquanto no caule provocou a redução dos teores de $\mathrm{Ca}$ e $\mathrm{S}$. Em plantas de milheto (Pennisetum glaucum L), cultivadas em solução nutritiva, Prado e Vidal (2008) observaram aumento no teor de K com a omissão de $\mathrm{P}$ e aumento dos teores de $\mathrm{N}$ e $\mathrm{P}$ com a omissão de K. Tais autores sugeriram que plantas sob deficiência de $\mathrm{K}$ ficam desequilibradas nutricionalmente. Soluções nutritivas desequilibradas causam problemas metabólicos nas plantas (FLORES et al., 2012), podendo levar aumento ou diminuição dos teores de nutrientes. Em pimenta- malagueta (Capsicum frutescens) cultivada em solução nutritiva, Flores et al. (2012) observaram aumentos nos teores de $\mathrm{Ne}$ redução dos teores de $\mathrm{Ca}$ nas raízes, com a omissão de K.

A omissão de Na reduziu os teores de $\mathrm{Na}$ no caule e de Ca nas raízes, e aumentou os teores de $\mathrm{N}$ e $\mathrm{S}$. A redução do teor de $\mathrm{Ca}$ devido à omissão de $\mathrm{Na}$ não era esperada, visto que ele compete com o Ca pelos mesmos sítios de absorção (SUBARAO et al., 2003). Entretanto, esse resultado é importante, pois evidencia que a ausência de $\mathrm{Na}$ foi prejudicial à absorção de Ca pela pupunheira.

As faixas de teores de macronutrientes e o $\mathrm{Na}$, que proporcionaram maior crescimento e aparentemente sem sintomas de deficiências, foram: 39,1 a 47,0 de $\mathrm{N} ; 3,55$ a 4,86 de P; 11 a 17,75 de K; 7,66 a 9,89 de Ca; 2,62 a 3,56 de $\mathrm{Mg} ; 2,03$ a 2,35 de 
$\mathrm{S}$, e 2,05 a 3,67 de Na, em $\mathrm{g} \mathrm{kg}^{-1}$. Em plantações de pupunheira, sob elevada taxa de fertilização, na Costa Rica, Deenik et al. (2000) citaram faixas adequadas de teores, em g kg-1, correspondes a: 25 a 40 para N; 1,5 a 3 para $\mathrm{P} ; 8$ a 15 para $\mathrm{K} ; 2$ a 5 para $\mathrm{Ca}$, e 2 a 3 para $\mathrm{Mg}$; entretanto, não indicaram valores para $\mathrm{S}$ e Na. Esses valores estão próximos aos encontrados no presente trabalho para o tratamento completo. Já Silva e Falcão (2002) sugerem como valores adequados: 30,7 para N; 3,4 para $\mathrm{P} ; 27,8$ para K; 12,7 para $\mathrm{Ca}$, e 3,5 para $\mathrm{Mg}$. Tal pesquisa indicou valores mais baixos para $\mathrm{N}$ e $\mathrm{P}$ e muito superiores para $\mathrm{K}$, o que pode estar relacionado à solução nutritiva utilizada, que não foi específica para espécies da família Arecaceae e cuja concentração de K na solução é superior.
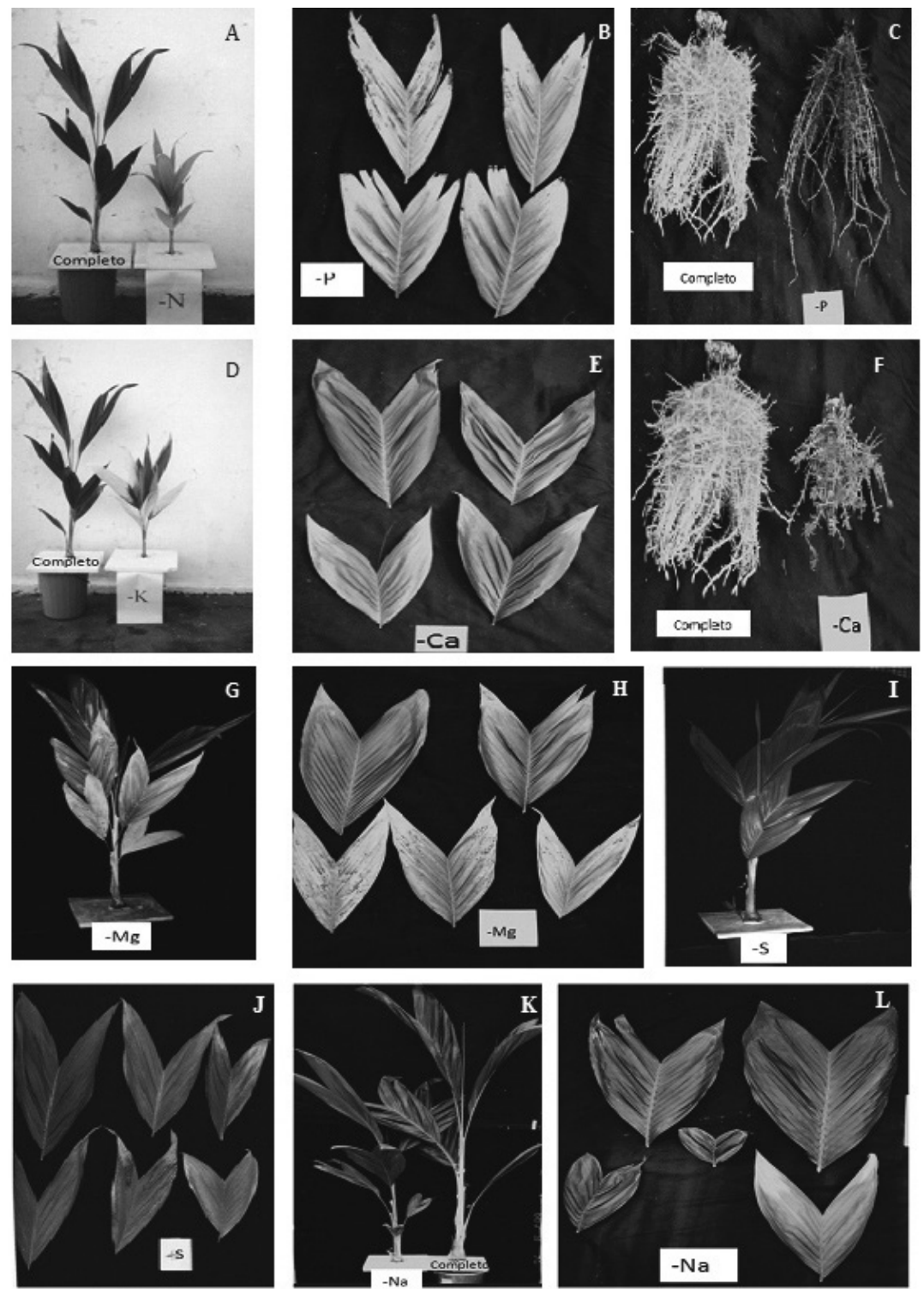

FIGURA 1 - Sintomas de deficiência de N (A), P (B, C), K (D), Ca (E, F), Mg (G, H), S (I, J) e Na (K, L) em mudas de pupunheira. 
TABELA 1 - Matéria seca das folhas, caule, raízes, parte aérea (PA), total, relação parte aérea/raiz (PA/R) e crescimento relativo (CR) de pupunheira em função da omissão de macronutrientes e Na.

\begin{tabular}{lccccccc}
\hline Tratamento & Folha & Caule & Raízes & PA & Total & PA/R & CR \\
\hline Completo & $66,5 \mathrm{a}$ & $70,5 \mathrm{a}$ & $62,3 \mathrm{a}$ & $137,0 \mathrm{a}$ & $199,3 \mathrm{a}$ & $2,19 \mathrm{~d}$ & 100,0 \\
$-\mathrm{N}$ & $8,5 \mathrm{e}$ & $6,5 \mathrm{~d}$ & $10,1 \mathrm{c}$ & $15,0 \mathrm{~d}$ & $25,1 \mathrm{~d}$ & $1,52 \mathrm{e}$ & 12,6 \\
$-\mathrm{P}$ & $19,7 \mathrm{de}$ & $8,1 \mathrm{~d}$ & $9,3 \mathrm{c}$ & $27,7 \mathrm{~d}$ & $37,0 \mathrm{~cd}$ & $3,00 \mathrm{~cd}$ & 18,6 \\
$-\mathrm{K}$ & $28,9 \mathrm{~cd}$ & $19,2 \mathrm{~cd}$ & $9,5 \mathrm{c}$ & $48,1 \mathrm{~cd}$ & $57,6 \mathrm{bcd}$ & $5,70 \mathrm{a}$ & 28,9 \\
$-\mathrm{Ca}$ & $45,3 \mathrm{bc}$ & $41,1 \mathrm{bc}$ & $25,2 \mathrm{~b}$ & $86,4 \mathrm{bc}$ & $111,6 \mathrm{~b}$ & $3,52 \mathrm{c}$ & 56,0 \\
$-\mathrm{Mg}$ & $37,2 \mathrm{~cd}$ & $29,3 \mathrm{~cd}$ & $15,9 \mathrm{~b}$ & $66,4 \mathrm{c}$ & $82,4 \mathrm{bc}$ & $4,76 \mathrm{~b}$ & 41,3 \\
$-\mathrm{S}$ & $56,4 \mathrm{ab}$ & $57,7 \mathrm{ab}$ & $52,7 \mathrm{a}$ & $114,1 \mathrm{ab}$ & $166,8 \mathrm{a}$ & $2,16 \mathrm{~d}$ & 83,7 \\
$-\mathrm{Na}$ & $57,3 \mathrm{ab}$ & $62,7 \mathrm{ab}$ & $58,2 \mathrm{a}$ & $120,0 \mathrm{ab}$ & $178,2 \mathrm{a}$ & $2,09 \mathrm{~d}$ & 89,4 \\
$\mathrm{CV}(\%)$ & 10,12 & 9,21 & 13,44 & 8,43 & 12,28 & 6,2 & \\
\hline
\end{tabular}

Médias seguidas pela mesma letra na coluna não diferem entre si, pelo teste de Tukey $(p>0,05)$.

TABELA 2 - Teor de N, P, K, Ca, Mg, S e Na presentes na matéria de seca da folha, caule e raiz de pupunheira em função da omissão de nutrientes.

\begin{tabular}{|c|c|c|c|c|c|c|c|c|c|c|c|c|}
\hline \multirow{2}{*}{ Tratamento } & $\mathrm{N}$ & $\mathrm{P}$ & & $\mathrm{K}$ & & $\mathrm{Ca}$ & & $\mathrm{Mg}$ & & $\mathrm{S}$ & & $\mathrm{Na}$ \\
\hline & \multicolumn{12}{|c|}{ Folhas $\left(\mathrm{g} \mathrm{kg}^{-1}\right)$} \\
\hline Completo & $39,10 \mathrm{~b}$ & 3,55 & $\mathrm{~b}$ & 11,00 & $\mathrm{~b}$ & 9,35 & $\mathrm{a}$ & 3,26 & $\mathrm{a}$ & 2,03 & $a b c$ & $2,05 \mathrm{ab}$ \\
\hline$-\mathrm{N}$ & $14,00 \mathrm{~d}$ & 4,35 & $\mathrm{a}$ & 14,74 & $a b$ & 9,89 & $\mathrm{a}$ & 3,04 & $\mathrm{a}$ & 2,31 & $\mathrm{ab}$ & $2,97 \mathrm{ab}$ \\
\hline$-\mathrm{P}$ & $39,80 \mathrm{~b}$ & 2,58 & $\mathrm{c}$ & 13,20 & $a b$ & 9,37 & $\mathrm{a}$ & 3,08 & $\mathrm{a}$ & 1,70 & $\mathrm{bc}$ & $3,08 \mathrm{ab}$ \\
\hline$-\mathrm{K}$ & $47,05 \mathrm{a}$ & 4,50 & $\mathrm{a}$ & 6,82 & $\mathrm{c}$ & 7,66 & $\mathrm{a}$ & 2,62 & $a b$ & 2,07 & $a b c$ & $2,20 \mathrm{ab}$ \\
\hline$-\mathrm{Ca}$ & $44,43 \mathrm{ab}$ & 4,86 & $\mathrm{a}$ & 17,75 & $\mathrm{a}$ & 4,59 & $\mathrm{~b}$ & 3,23 & $\mathrm{a}$ & 2,50 & $\mathrm{a}$ & 3,67 a \\
\hline$-\mathrm{Mg}$ & $41,40 \mathrm{ab}$ & 3,99 & $a b$ & 15,40 & $\mathrm{ab}$ & 9,85 & $\mathrm{a}$ & 1,52 & $\mathrm{~b}$ & 2,23 & $a b$ & 3,52 a \\
\hline$-\mathrm{S}$ & $30,23 \mathrm{c}$ & 3,97 & $a b$ & 11,88 & $\mathrm{~b}$ & 9,07 & $\mathrm{a}$ & 3,07 & $\mathrm{a}$ & 1,50 & $\mathrm{c}$ & $3,15 \mathrm{ab}$ \\
\hline$-\mathrm{Na}$ & $41,60 \mathrm{ab}$ & 4,62 & $\mathrm{a}$ & 15,84 & $a b$ & 9,30 & $\mathrm{a}$ & 3,56 & $\mathrm{a}$ & 2,35 & $\mathrm{a}$ & $1,87 \mathrm{~b}$ \\
\hline \multirow[t]{2}{*}{$\mathrm{CV}(\%)$} & 21,76 & 11,65 & & 14,3 & & 7,81 & & 12,45 & & 10,87 & & 11,14 \\
\hline & \multicolumn{12}{|c|}{ Caule $\left(\mathrm{g} \mathrm{kg}^{-1}\right)$} \\
\hline Completo & $22,10 \mathrm{ab}$ & 5,14 & $\mathrm{a}$ & 16,28 & $\mathrm{c}$ & 11,04 & $\mathrm{ab}$ & 3,73 & $\mathrm{bc}$ & 2,22 & $\mathrm{~b}$ & 3,30 a \\
\hline$-\mathrm{N}$ & $8,80 \mathrm{c}$ & 4,10 & $\mathrm{bc}$ & 20,46 & $\mathrm{a}$ & 7,10 & $\mathrm{c}$ & 2,97 & $\mathrm{~cd}$ & 2,13 & $\mathrm{~b}$ & $1,65 \mathrm{bc}$ \\
\hline$-\mathrm{P}$ & $22,70 \mathrm{ab}$ & 2,50 & $\mathrm{c}$ & 18,92 & $a b$ & 8,33 & bc & 3,44 & $\mathrm{~cd}$ & 1,99 & $\mathrm{~b}$ & $1,76 \mathrm{bc}$ \\
\hline$-K$ & $27,00 \mathrm{a}$ & 4,10 & $a b c$ & 11,88 & d & 8,17 & $\mathrm{c}$ & 3,72 & bcd & 1,52 & $\mathrm{~cd}$ & $1,87 \mathrm{bc}$ \\
\hline$-\mathrm{Ca}$ & $21,26 \mathrm{ab}$ & 5,46 & $\mathrm{a}$ & 17,74 & $\mathrm{bc}$ & 5,00 & d & 4,87 & $\mathrm{ab}$ & 2,46 & $a b$ & $2,57 \mathrm{ab}$ \\
\hline$-\mathrm{Mg}$ & $21,46 \mathrm{ab}$ & 5,28 & $\mathrm{a}$ & 17,74 & $\mathrm{bc}$ & 11,79 & $\mathrm{a}$ & 2,55 & $\mathrm{~d}$ & 2,34 & $a b$ & $2,42 \mathrm{abc}$ \\
\hline$-\mathrm{S}$ & $21,33 \mathrm{ab}$ & 4,58 & $a b$ & 17,89 & $\mathrm{bc}$ & 8,50 & $\mathrm{bc}$ & 4,13 & $\mathrm{bc}$ & 1,14 & $\mathrm{~d}$ & $1,91 \mathrm{abc}$ \\
\hline$-\mathrm{Na}$ & $17,20 \mathrm{~b}$ & 5,72 & $\mathrm{a}$ & 17,82 & $\mathrm{bc}$ & 11,69 & $\mathrm{a}$ & 5,27 & $\mathrm{a}$ & 2,93 & $\mathrm{a}$ & $1,03 \mathrm{c}$ \\
\hline \multirow[t]{2}{*}{$\mathrm{CV}(\%)$} & 8,09 & 13,31 & & 12,7 & & 4,79 & & 17,87 & & 9,33 & & 12,76 \\
\hline & \multicolumn{12}{|c|}{ Raiz $\left(\mathrm{g} \mathrm{kg}^{-1}\right)$} \\
\hline Completo & $22,40 \mathrm{c}$ & 5,41 & $a b$ & 15,18 & $a b$ & 9,75 & $\mathrm{a}$ & 2,78 & $\mathrm{a}$ & 2,38 & $\mathrm{c}$ & $0,88 \mathrm{ab}$ \\
\hline$-\mathrm{N}$ & $7,65 \mathrm{e}$ & 5,82 & $\mathrm{a}$ & 10,12 & $\mathrm{bc}$ & 3,77 & $\mathrm{bc}$ & 1,35 & $\mathrm{~b}$ & 2,83 & $\mathrm{bc}$ & $0,77 \mathrm{ab}$ \\
\hline$-\mathrm{P}$ & $15,90 \mathrm{~d}$ & 3,28 & $\mathrm{c}$ & 11,66 & $a b c$ & 4,60 & $\mathrm{~b}$ & 1,66 & $a b$ & 2,62 & $\mathrm{bc}$ & $0,88 \mathrm{ab}$ \\
\hline$-\mathrm{K}$ & $27,15 \mathrm{ab}$ & 6,23 & $\mathrm{a}$ & 7,26 & $\mathrm{c}$ & 3,76 & $\mathrm{bc}$ & 1,48 & $\mathrm{~b}$ & 3,20 & $a b$ & $0,99 \mathrm{ab}$ \\
\hline$-\mathrm{Ca}$ & $30,43 \mathrm{a}$ & 4,10 & $\mathrm{bc}$ & 12,03 & $a b c$ & 2,26 & $\mathrm{c}$ & 1,36 & $\mathrm{~b}$ & 3,13 & $\mathrm{~b}$ & $1,32 \mathrm{a}$ \\
\hline$-\mathrm{Mg}$ & $26,60 \mathrm{abc}$ & 4,96 & $a b$ & 9,68 & $\mathrm{bc}$ & 4,19 & $\mathrm{bc}$ & 0,95 & $\mathrm{~b}$ & 3,44 & $\mathrm{a}$ & $1,25 \mathrm{a}$ \\
\hline$-\mathrm{S}$ & $24,17 \mathrm{bc}$ & 5,32 & $a b$ & 14,81 & $a b$ & 4,11 & $\mathrm{bc}$ & 1,69 & $a b$ & 1,88 & $\mathrm{~d}$ & 1,32 a \\
\hline$-\mathrm{Na}$ & $28,27 \mathrm{ab}$ & 4,99 & $a b$ & 17,31 & $\mathrm{a}$ & 4,03 & $\mathrm{bc}$ & 1,61 & $a b$ & 3,47 & $\mathrm{a}$ & $0,59 \mathrm{~b}$ \\
\hline CV (\%) & 13,1 & 14,52 & & 11,34 & & 8,60 & & 10,9 & & 16,75 & & 17,65 \\
\hline
\end{tabular}

Médias seguidas pela mesma letra na coluna não diferem entre si, pelo teste de Tukey $(\mathrm{p}>0,05)$. 
TABELA 3 - Acúmulo de N, P, K, Ca, Mg, S e Na presentes na matéria seca de folha, caule, raiz e total de mudas de pupunheira.

\begin{tabular}{|c|c|c|c|c|c|c|c|}
\hline \multirow{2}{*}{ Tratamento } & $\mathrm{N}$ & $\mathrm{P}$ & $\mathrm{K}$ & $\mathrm{Ca}$ & $\mathrm{Mg}$ & $\mathrm{S}$ & $\mathrm{Na}$ \\
\hline & \multicolumn{7}{|c|}{ Folha (mg/planta) } \\
\hline Completo & $2607,02 \mathrm{a}$ & $234,86 a b$ & $728,13 a$ & $614,22 \mathrm{a}$ & $214,19 a$ & $134,65 \mathrm{ab}$ & $133,21 \mathrm{ab}$ \\
\hline$-\mathrm{N}$ & $118,90 \mathrm{e}$ & $36,74 \mathrm{e}$ & $125,76 \mathrm{~d}$ & $84,90 \mathrm{~d}$ & $26,25 \mathrm{c}$ & $19,49 d$ & $25,70 \mathrm{c}$ \\
\hline$-\mathrm{P}$ & $784,17 \mathrm{de}$ & $51,02 \mathrm{de}$ & $262,40 \mathrm{bcd}$ & $185,02 d$ & $60,93 \mathrm{c}$ & $33,86 \mathrm{~cd}$ & $61,43 b c$ \\
\hline$-K$ & $1362,85 \mathrm{~cd}$ & 132,11 cde & $194,69 \mathrm{~cd}$ & $219,83 \mathrm{~cd}$ & $74,49 \mathrm{c}$ & $60,01 \mathrm{~cd}$ & $62,78 \mathrm{bc}$ \\
\hline$-\mathrm{Ca}$ & $2014,51 \mathrm{abc}$ & $224,00 \mathrm{abc}$ & $799,48 \mathrm{a}$ & $206,50 \mathrm{~cd}$ & $144,78 b$ & $112,05 \mathrm{ab}$ & $167,01 \mathrm{a}$ \\
\hline$-\mathrm{Mg}$ & $1521,97 \mathrm{~cd}$ & $145,09 \mathrm{bc}$ & $576,41 \mathrm{abc}$ & $365,43 b c$ & $57,43 \mathrm{c}$ & $84,11 b c$ & $134,58 b c$ \\
\hline$-S$ & $1699,23 \mathrm{~cd}$ & $224,33 a b$ & $660,05 \mathrm{ab}$ & $506,59 \mathrm{ab}$ & $171,09 \mathrm{ab}$ & $84,43 a b c$ & $179,42 \mathrm{a}$ \\
\hline$-\mathrm{Na}$ & $2370,53 \mathrm{ab}$ & $262,31 \mathrm{a}$ & $922,87 \mathrm{a}$ & $533,91 \mathrm{a}$ & $205,17 \mathrm{ab}$ & $135,90 \mathrm{a}$ & $107,75 \mathrm{ab}$ \\
\hline \multirow[t]{2}{*}{ CV (\%) } & 14,1 & 23,4 & 27,7 & 15,3 & 12,9 & 13,6 & 12,3 \\
\hline & \multicolumn{7}{|c|}{ Caule (mg/planta) } \\
\hline Completo & $1601,90 a$ & $369,05 a$ & $1142,35 \mathrm{a}$ & $786,10 \mathrm{a}$ & $262,37 \mathrm{ab}$ & $157,99 a$ & $240,08 \mathrm{a}$ \\
\hline$-\mathrm{N}$ & $57,56 \mathrm{c}$ & $21,14 d$ & $133,11 \mathrm{e}$ & $46,21 \mathrm{e}$ & $19,40 \mathrm{c}$ & $13,84 d$ & $10,70 \mathrm{c}$ \\
\hline$-\mathrm{P}$ & $182,68 \mathrm{c}$ & $20,24 d$ & $152,39 \mathrm{e}$ & $67,24 \mathrm{e}$ & $27,69 \mathrm{c}$ & $16,05 \mathrm{~d}$ & $14,25 \mathrm{c}$ \\
\hline$-\mathrm{K}$ & $526,95 \mathrm{bc}$ & $79,10 \mathrm{~cd}$ & $228,89 \mathrm{de}$ & $159,80 \mathrm{de}$ & $72,28 \mathrm{c}$ & $29,33 \mathrm{~cd}$ & $37,22 b c$ \\
\hline$-\mathrm{Ca}$ & $874,46 a b c$ & 221,69abc & $729,91 \mathrm{bc}$ & $205,02 \mathrm{de}$ & $200,36 b$ & $100,69 b$ & $102,07 b$ \\
\hline$-\mathrm{Mg}$ & $614,82 \mathrm{bc}$ & $153,04 d$ & $519,36 \mathrm{~cd}$ & $344,14 \mathrm{~cd}$ & $74,33 \mathrm{c}$ & $67,95 \mathrm{bc}$ & $68,84 b$ \\
\hline$-S$ & $1247,02 \mathrm{ab}$ & $260,48 \mathrm{ab}$ & $1028,69 \mathrm{ab}$ & $485,14 b c$ & $234,84 b$ & $65,50 \mathrm{bc}$ & $107,54 b$ \\
\hline$-\mathrm{Na}$ & $1090,13 \mathrm{ab}$ & $358,07 \mathrm{a}$ & $1117,14 \mathrm{a}$ & $733,26 a b$ & $327,43 a$ & $182,26 \mathrm{a}$ & $64,62 b c$ \\
\hline \multirow[t]{2}{*}{ CV (\%) } & 16 & 21 & 17,6 & 19,4 & 25,7 & 12,2 & 9,7 \\
\hline & \multicolumn{7}{|c|}{ Raiz (mg/planta) } \\
\hline Completo & $1373,70 \mathrm{a}$ & $332,48 \mathrm{a}$ & $956,76 a$ & $618,30 \mathrm{a}$ & $179,19 a$ & $149,36 \mathrm{ab}$ & $56,09 \mathrm{ab}$ \\
\hline$-\mathrm{N}$ & $77,27 \mathrm{~d}$ & $58,98 \mathrm{bc}$ & $101,93 b c$ & $41,16 \mathrm{c}$ & $13,93 \mathrm{c}$ & $28,95 \mathrm{c}$ & $7,92 \mathrm{c}$ \\
\hline$-\mathrm{P}$ & $147,24 d$ & $30,49 c$ & $109,58 \mathrm{bc}$ & $42,65 c$ & $15,54 \mathrm{c}$ & $24,51 \mathrm{c}$ & $8,27 \mathrm{c}$ \\
\hline$-\mathrm{K}$ & $258,87 \mathrm{~cd}$ & $58,65 \mathrm{bc}$ & $68,05 \mathrm{c}$ & $37,13 \mathrm{c}$ & $13,97 \mathrm{c}$ & $29,84 \mathrm{c}$ & $9,70 \mathrm{c}$ \\
\hline$-\mathrm{Ca}$ & $776,81 \mathrm{bc}$ & $101,85 b$ & $312,95 b$ & $57,01 \mathrm{c}$ & $34,42 b c$ & $79,74 \mathrm{bc}$ & $33,41 b c$ \\
\hline$-\mathrm{Mg}$ & $414,19 \mathrm{~cd}$ & $76,67 \mathrm{bc}$ & $157,40 \mathrm{bc}$ & $60,60 c$ & $15,71 \mathrm{c}$ & $56,27 \mathrm{c}$ & $20,32 b c$ \\
\hline$-S$ & $1273,32 \mathrm{ab}$ & $280,53 a$ & $779,67 \mathrm{a}$ & $216,93 b$ & $89,18 \mathrm{ab}$ & $99,27 b c$ & $69,87 \mathrm{a}$ \\
\hline$-\mathrm{Na}$ & $1647,12 \mathrm{a}$ & $291,82 \mathrm{a}$ & $1005,66 a$ & $218,62 b$ & $93,39 \mathrm{ab}$ & $203,11 \mathrm{a}$ & $34,75 \mathrm{abc}$ \\
\hline \multirow[t]{2}{*}{ CV (\%) } & 9,3 & 13,4 & 26,8 & 11,3 & 19,7 & 22,3 & 20,1 \\
\hline & \multicolumn{7}{|c|}{ Total (mg/planta) } \\
\hline Completo & $5582,62 \mathrm{a}$ & $936,39 a$ & $2827,24 \mathrm{ab}$ & $2018,61 \mathrm{a}$ & $655,75 \mathrm{a}$ & 441,99ab & $429,38 \mathrm{a}$ \\
\hline$-\mathrm{N}$ & $253,72 \mathrm{f}$ & $116,86 \mathrm{c}$ & $360,81 \mathrm{~d}$ & $172,27 \mathrm{e}$ & $59,58 \mathrm{c}$ & $62,28 \mathrm{e}$ & $44,32 \mathrm{e}$ \\
\hline$-\mathrm{P}$ & $1114,09 \mathrm{ef}$ & $101,75 \mathrm{c}$ & $524,37 d$ & 294,91de & $104,16 \mathrm{c}$ & $74,42 \mathrm{e}$ & $83,95 \mathrm{de}$ \\
\hline$-\mathrm{K}$ & $2148,67 \mathrm{de}$ & $269,86 \mathrm{~cd}$ & $491,62 d$ & $416,76 \mathrm{de}$ & $160,75 c$ & $119,18 \mathrm{de}$ & $109,69 \mathrm{de}$ \\
\hline$-\mathrm{Ca}$ & $3665,78 \mathrm{bcd}$ & $547,55 \mathrm{bc}$ & $1842,33 \mathrm{bc}$ & $468,53 \mathrm{de}$ & $379,56 b$ & $292,48 b c$ & $302,49 a b c$ \\
\hline$-\mathrm{Mg}$ & $2550,97 \mathrm{cde}$ & $374,80 \mathrm{~cd}$ & $1253,17 \mathrm{~cd}$ & $770,16 \mathrm{~cd}$ & $147,47 \mathrm{c}$ & 208,33 cde & $223,74 d$ \\
\hline$-\mathrm{S}$ & $4219,57 \mathrm{abc}$ & $765,34 \mathrm{a}$ & $2468,41 \mathrm{ab}$ & $1208,66 b c$ & $495,11 \mathrm{ab}$ & $249,20 \mathrm{~cd}$ & $356,84 a b$ \\
\hline$-\mathrm{Na}$ & $5107,78 \mathrm{ab}$ & $912,21 \mathrm{a}$ & $3045,67 \mathrm{a}$ & $1485,78 \mathrm{ab}$ & $625,99 a$ & $521,28 \mathrm{e}$ & $207,12 \mathrm{~cd}$ \\
\hline CV $(\%)$ & 13,14 & 21,23 & 24,87 & 20,05 & 17,91 & 12,4 & 31,12 \\
\hline
\end{tabular}

Médias seguidas pela mesma letra na coluna não diferem entre si, pelo teste de Tukey $(p>0,05)$. 


\section{CONCLUSÕES}

1-As omissões de macronutrientes em pupunheira provocam sintomas visuais de deficiência nutricionais comuns às outras espécies. A ausência de Na causa clorose e necrose das ponteiras das folhas.

2-Os elementos que mais restringem a produção de matéria seca são o N, P e K. A omissão de $\mathrm{Na}$ reduz em $10,6 \%$ a matéria seca.

3-Os teores e acúmulo dos elementos em pupunheira seguem a ordem $\mathrm{N}>\mathrm{K}>\mathrm{Ca}>\mathrm{P}>\mathrm{Mg}>\mathrm{S}>\mathrm{Na}$.

\section{REFERÊNCIAS}

ANANDACOOMARASWAMY, A.; De COSTA, W. A. J. M.; TENNAKOON, P. K.; VANDERWERF, A. The physiological basis of increased biomass partitioning to roots upon nitrogen deprivation in young clonal tea (Camellia sinensis (L.) Kuntz). Plant and Soil, Dordrecht, v. 238, p. 1-9, 2002.

ARES, A.; FLACÃO, N.; YUYAMA, K.; YOST, R. S.; CLEMENT, C. R. Response to fertilization and nutrient deficiency diagnostics in peach palm in central Amazonia. Nutrient Cycling in Agroecossystems, Dordrecht, v. 66, p. 221-232, 2003.

ARMENGUAD, P.; SULPICE, R.; MILLER, A. J.; STITT, M.; AMTMANN, A.; GIBON, Y. Multilevel analysis of primary metabolism provides new insights into the role of potassium nutrition for glycolysis and nitrogen assimilation in Arabidopsis roots. Plant Physiology, Bethesda, v. 150, p. 772 $-785,2009$.

BELLAMINE, J.; PENEL, C.; GREEPIN, H.; GASPAR, T. Confirmation of the role of auxin and calcium in the late phases of adventitious root formation. Plant Growth Regulation, Dordrecht, v. 26, p. $191-194,1998$.

CUNHA, A. C. M.; PAIVA, H. N.; XAVIER, A.; OTONI, W. C. Papel da nutrição mineral na formação de raízes adventícias em plantas lenhosas. Pesquisa Florestal Brasileira, Colombo, n. 58, 2009.
DEENIK, A.; ARES, A.; YOST, R.S. Fertilization response and nutrient diagnosis in peach palm (Bactrisgasipaes): a review. Nutrient Cycling in Agroecosystems, Dordrecht, v. 56, p.195-207, 2000.

DUFOUR, F.; QUENCEZ, P.; SCHMITTI, G. Technique de culture en solutions nutritive du palmier à huileet du cocotier. Oléagineux, Paris, v.33, n. 10, p.485-490, 1978.

FERNANDES, A. R.; CARVALHO, J. G. Crescimento de mudas de pupunheira (Bactris gasipaesH.B.K.) em função de relações do K com o $\mathrm{Ca}$ e com o $\mathrm{Na}$, em solução nutritiva. Cerne, Lavras, v. 7, n. 1, p. 84-89, 2001.

FERNANDES, A. R.; CARVALHO, J. G.; CURI, N.; PINTO, J. E. B. P.; GUIMARÃES, P. T. G. 2002. Nutrição mineral de mudas de pupunheira sob diferentes níveis de salinidade. Pesquisa Agropecuária Brasileira, Brasília, v. 37, n. 11, p. 1.613-1.619, 2002.

FLORES, R. A.; ALMEIDA, T. B. F.; POLITI, L. S.; PRADO, R. de M.; JOSÉ C.; BARBOSA, J. C. Crescimento e desordem nutricional em pimenteira-malagueta cultivada em soluções nutritivas suprimidas de macronutrientes. Revista Brasileira de Ciências Agrárias, Recife, v. 7, n. 1, p. 104-110, 2012.

FITA, A.; NUEZ, F.; PICÓ, B. Diversity in root architecture and response to $\mathrm{P}$ deficience in seedlings of Cucumis melo L. Euphytica, Wageningen, v. 181, n. 3, p. 323-339, 2011.

FREITAS, M. S. M.; MONNERAT, P. H.; CARVALHO, A. J. C.; VASCONCELLOS, M. A. $\mathrm{S}$. Sintomas visuais de deficiência de macronutrientes e boro em maracujazeiro-doce. Revista Brasileira de Fruticultura, Jaboticabal, v. 33, n. 4, p. 13291341, 2011.

HAWKESFORD, M. J. Plant response to sulphur deficiency and the genetic manipulation of sulphate transporters to improve S-utilization efficiency. Journal of Experimental Botany, Oxford, v. 51, p. 131-138, 2000.

HETHERINGTON, A. M.; BROWNLEE, C. The generation of $\mathrm{Ca}^{+}$signals in plants. Annual Review of Plant Biology, Palo Alto, v. 55, p. 401-427, 2004. 
HORTENSTEINER, S.; FELLER, U. Nitrogen metabolism and remobilization during senescence. Journal of Experimental Botany, Oxford, v. 53, p. $927-937,2002$.

KANAI, S.; OHKURA, K.; ADU-GYAMF, J. J.; MOAPATRA, P. K.; NRGUYEN, N. T.; SANEOKA, H.; FUJITA, K. Depression of sink activity precedes the inhibition of biomass production in tomato plants subjected to potassium deficiency stress. Journal of Experimental Botany, Oxford, v. 58, p. 2917 2928, 2007.

KARTHIKEYAN, A. S.; VARADARAJAN, D. K.; JAIN, A.; HELD, M. A.; CARPITA, N. C. Phosphate starvation responses are mediated by sugar signaling in Arabdopisis. Planta, Berlin, v. 220, p. 219-225, 2007.

LAVIOLA, B. G.; DIAS, L. A. S.Teor e acúmulo de nutrientes em folhas e frutos de pinhão-manso. Revista Brasileira de Ciência do Solo, Viçosa, MG, v. 32, n. 5, p. 1969-1975, 2008.

LÓPEZ-BUCIO J.; CRUZ-RAMÍREZ A.; HERRERA-ESTRELLA L. The role of nutrient availability in regulating root architecture. Current Opinion in Plant Biology. v.6, p.280-287, 2003.

MARQUES, T.C.L.L.S.M.; CARVALHO, J.G.; LACERDA, M.P.C; MOTA, P.E.F. Crescimento inicial do paricá (Schizolobium amazonicum) sob omissão de nutrientes e de sódio em solução nutritiva. Cerne, Lavras, v. 10, n. 2, p.184-195, 2004.

MALAVOLTA, E. Manual de nutrição mineral de plantas. São Paulo: Ceres, 2006. 638 p.

MIRANDA, R. S.; SUDERIO, F. B.; SOUSA, A. F.; GOMES FILHO, E. Deficiência nutricional em plântulas de feijão-de-corda decorrente da omissão de macro e micronutrientes. Revista Ciência Agronômica, Fortaleza, v. 41, n. 3, p. 326-333, 2010.

PAUL, M.J.; DRISCOLL, S.P. Sugar repression of photosynthesis: the role of carbohydrates in signaling nitrogen deficiency through source sink-imbalance. Plant Cell and Environment, Malden, v. 20, p. 110-116, 1997.
PILON-SMITS, E. A. H.; QUINN, C.F; W.; TAPKEN, W.; MALAGONI, M.; SCHIAVON,M. Physiological functions of beneficial elements. Current Opinion in Plant Biology, Cambridge, v. 12, p. 267-274, 2009.

PRADO, R. de M.; ROMUALDO, L. M.; ROZANE, D. E. Omissão de macronutrientes no desenvolvimento e no estado nutricional de plantas de sorgo (cv. BRS 3010) cultivadas em solução nutritiva. Científica, Jaboticabal, v. 35, n. 2, p. 122 - 128, 2007.

PRADO, R. de M.; VIDAL, A. A. Efeitos da omissão de macronutrientes em solução nutritiva sobre o crescimento e a nutrição do milheto. Pesquisa Agropecuária Tropical, Goiânia, v. 38, n. 3, p. 208-214, 2008.

RICHARD-MOLARD, C.; KRAPP, A.; BRUN, F.; NEY, B.; DANIEL-VEDELE, F.; CHAILLOU, S. Plant response to nitrate starvation is determined by nitrogen capacity matched by nitrate uptake capacity in two Arabidopsis genotypes. Journal of Experimental Botany, Oxford, v. 59, 779-791, 2008.

SARCINELLI, T. S.; RIBEIRO JÚNIOR, E. S.; DIAS, L. E.; LYNCH, L. S. Sintomas de deficiência nutricional em mudas de Acácia holosericea em resposta à omissão de macronutrientes. Revista Árvore, Viçosa, MG, v. 28, n. 2, p. 173-181, 2004.

SCHACHTMAN, D. P.; REID, R. J.; AYLING, S. M. Phosphorus uptake by plants: from soil to cell. Plant Physiology, Bethesda, v. 116, p. 447-453, 1998.

SHAUL, O. Magnesium transport and function in plants: the tip of the iceberg. Biometals, London, v. 25 , p. $309-323,2002$

SILVA, J. R. A.; FALCÃO, N. P. S. Caracterização de sintomas de carências nutricionais em mudas de pupunheira cultivada em solução nutritiva. Acta Amazônica, Manaus, v. 32, n. 4, p. 529-539, 2002.

SOUSA, H. U.; RAMOS, J. D.; CARVALHO, J. G.; FERREIRA, E. A. Nutrição de mudas de açaizeiro sob relações cálcio:potássio:sódio em solução nutritiva. Ciência Agrotécnica, Lavras, v. 28, n. 1, p. $56-62.2004$. 
SUBBARAO, G. V.; ITO, O.; BERRY W.L.; WHEELER, R. M. Sodium: a functional plant nutrient. Critical Reviews in Plant Sciences, Apopka, v. 22, p. 391-416, 2003.

VIÉGAS, I. J. M.; MEIRELES, R. O.; FRAZÃO, D. A. C.; CONCEIÇÃO, H. E. O. Avaliação da fertilidade de um Latossolo Amarelo textura média para o cultivo do açaizeiro no Estado do Pará. Revista de Ciências Agrárias, Belém, n. 52, p. 23-36, 2009.

VIÉGAS; I. J. M.; FRAZÃO, D. A. C.; THOMAZ, M. A. A.; CONCEIÇÃO; HERÁCLITO, E. O.; PINHEIRO, E. Limitações nutricionais para o cultivo de açaizeiro em Latossolo Amarelo textura média, Estado do Pará. Revista Brasileira de Fruticultura, Jaboticabal, v.26, n. 2, p. 382-384, 2004.
WHITE, J. E.; BROADLEY, M. R. Calcium in plants. Annals of Botany, London, v.92, p. 487-511, 2003.

YUYAMA, K.; CHÁVEZ, W. B.; PEREIRA, B. G.; SILVA, I. A. Efeito da densidade de plantas e da adubação NPK na produção inicial de palmito de pupunheira. Revista Brasileira de Ciência do Solo, Viçosa, MG, v. 29, n. 3, p. 373-378, 2005. 